\title{
Possibilities and Limitations of Scanning Electrochemical Microscopy of Mg and Mg alloys
}

\author{
S. Thomas ${ }^{1}$, J. Izquierdo ${ }^{2}$, N. Birbilis ${ }^{1}$, R. M. Souto ${ }^{2,3}$ \\ ${ }^{1}$ Department of Materials Engineering, Monash University, Clayton, Victoria, 3800, Australia \\ ${ }^{2}$ Department of Chemistry, University of La Laguna, P.O. Box 456, E-38200 La Laguna, Tenerife, \\ Canary Islands, Spain \\ ${ }^{3}$ Instituto Universitario de Materiales y Nanotecnologías, University of La Laguna, E-38200 La Laguna, \\ Tenerife, Canary Islands, Spain
}

\begin{abstract}
:
Magnesium (Mg) and its alloys undergo corrosion at high rates in aqueous electrolytes of essentially any concentration, releasing copious amounts of hydrogen into the electrolyte, thereby making conventional SECM measurements difficult upon Mg. A few examples of the issues associated with Mg SECM, and strategies to counter these issues are discussed in this review. SECM has been employed in Sample Generation-Tip Collection (SG-TC) mode to image cathodic sites on the Mg surface, wherein, the Pt tip oxidises hydrogen emanating from the specimen surface. This mode of SECM does not clearly reveal a close correspondence between the $\mathrm{Mg}$ microstructure and its associated electrochemical reactivity; the hydrogen currents measured by the Pt tip found to vary by over an order of magnitude with time. Convective fluxes due to hydrogen bubbles, local acidification arising from protons released during hydrogen oxidation on the Pt tip, and the negative different effect of $\mathrm{Mg}$ may contribute to these high tip currents. SECM has also been performed on $\mathrm{Mg}$ using $\mathrm{Mg}^{2+}$ ion selective electrodes (ISEs), where an $\mathrm{Mg}^{2+}$ ionophore cocktail serves as a $\mathrm{Mg}^{2+}$ ion permeable membrane. The $\mathrm{Mg}^{2+}$ ions diffuse/migrate through the membrane which eventually settles at the equilibrium membrane (Donnan) potential. This potential varies with the logarithm of $\mathrm{Mg}^{2+}$ ion concentration in the electrolyte, and thus could be used to ascertain the local $\mathrm{Mg}^{2+}$ ion concentrations (or anodic sites) on the surface. This technique needs to be complimented with other modes of SECM to obtain holistic electrochemical micrographs of a corroding Mg surface.
\end{abstract}

Keywords: Magnesium, SECM, hydrogen evolution, bubbles, ion-selective electrodes 


\section{Introduction:}

Magnesium and its alloys have attracted great interest for potential deployment in light-weight structural applications [1]. One key drawback of $\mathrm{Mg}$ alloys is that they are highly reactive in aqueous environments, and thus prone to multiple forms of corrosion including spontaneous dissolution, galvanic coupling and localised corrosion [2]. The corrosion potentials of pure Mg and most Mg alloys are well below -1.5 VSCE [3-4], thus permitting a high rate of spontaneous hydrogen evolution reaction (HER) upon the Mg surface [5]. For Mg alloys, the HER predominantly takes place on secondaryphases or metallic impurities dispersed in the Mg matrix [6-9]. The microstructure of an $\mathrm{Mg}$ alloy (including the matrix, secondary phases and impurity sites), should correlate with the kinetics of the HER on Mg, at least initially. This spatial relationship has been shown by several researchers, wherein, the microstructure is analysed using SEM and correlated with corrosion damage [7-9]. For direct comparison, a local electrochemical technique must be appropriately exploited to accurately probe the relationship between the $\mathrm{Mg}$ alloy microstructure and its corrosion. If this is not possible, then the utility of a scanning electrochemical technique is limited. To electrochemically image a corroding surface in an aqueous solution as it experiences corrosion, methods such as the Scanning Vibrating Electrode Technique (SVET), Scanning Electrochemical Microscopy (SECM) and even Scanning IonSelective Electrode Technique (SIET) are some electrochemical techniques which can decipher local anode and cathode sites in a corroding system [10-17]. The SECM method, which is the focus of this review, can monitor local changes in conductivity, composition, potentials and rates with high spatial resolution, and thus is extensively used in physicochemical, biomedical studies and to electrochemically image corroding metals [13-17]. The technique has been described in the next section. In order to be useful in the study of Mg and its alloys, SECM would need to be capable of offering insights not possible by other methods, such as SVET that has been recently shown to offer significant information on the temporal evolution of Mg corrosion.

\section{Scanning Electrochemical Microscopy (SECM)}

In this technique, an ultramicroelectrode (UME), also known as the tip, constitutes the sensing probe, though occasionally may be used to induce changes in the investigated substrate [18]. The tip and the substrate are positioned within an electrochemical cell along with a reference and counter electrodes. The use of a bipotentiostat allows both the tip and the substrate to act as independently-controlled working electrodes, though the substrate may also be left unbiased, nominally at its open circuit corrosion potential in the environment when naturally corroding conditions are explored. The UME 
(tip) is capable of moving in $X-Y-Z$ coordinates, and thus can raster the substrate surface at any prescribed height. Changes in the probe response when located in the proximity of the substrate are related to local solution composition, the tip-substrate distance and the electrochemical/electrical activity of the substrate [19]. An image of the spatial electrochemical reactions on a sample can thus be obtained by recording/analysing the tip response, as the tip is rastered across the substrate surface. This is because the electrochemical response of the probe experience perturbations when in close proximity with the substrate surface, therefore enabling one to record an electrochemical micrograph of the surface [19].

UMEs can be classified into either amperometric and potentiometric probes depending on their operation principles and applications. In the former, active polarizable microelectrodes of noble metals or carbon are employed to monitor faradaic currents due to electrochemical conversion of an electroactive species. The most frequent probe geometry consists of a disc of the material sealed in some insulating material (frequently glass), offering a disc-shaped active surface typically in the micrometer or submicrometer ranges [20]. Potentiometric operation is achieved using ion-selective microelectrodes (ISME) instead [21]. In this case, the potential sensed at the probe is a function of the local concentration of a relevant ionic species present in the electrolyte. Furthermore, dual amperometric/potentiometric probes are obtained by using materials that exhibit a dual function in different potential ranges [22], namely amperometrically when the material is in its metallic state, whereas the potential of the oxide-coated metal surface responds to changes in the $\mathrm{pH}$ of the environment.

Finally, in addition to the amperometric and potentiometric operation modes for the collection of electrochemical data in SECM, a third group is configured by the AC modes in SECM, namely frequency-dependent alternating-current SECM (AC-SECM) [23], and the more recent introduction of the Scanning Electrochemical Impedance Microscopy (SEIM) [24]. These methods have in common that an alternating potential signal is applied to the UME instead of a constant potential, whereas an alternating current of the same frequency is monitored to derive the electrochemical reactivity of the substrate. Differences in surface conductivity related to differences in electrochemical reactivity on the substrate can be detected by either displaying the alternating current response as a function of tip positioning for chemical imaging purposes as in AC-SECM, or it can be used to derive the local impedance response in SEIM.

\section{The electrochemistry of the UME}


In conventional SECM, an active micrometer-sized electrode is used to electrochemically image the substrate surface under amperometric operation [19,25]. The Pt UME probe consists of a Pt microdisk embedded in an insulating sheath, usually glass. The ratio of the radius of the insulating sheath, to the radius of the active Pt UME surface (with both measured from the centre of the microdisk) is defined as the RG ratio. When RG is greater than 10, a hemispherical diffusion profile of the electroactive species in the solution envelopes the microdisk electrode surface; with the disk surface at the vertex of the hemisphere oxidising/reducing the electroactive species. However, if the RG is less than 10 the diffusion profile will extend behind the surface of the UME and thus in practice, UMEs with RG ratio greater than 10 are nominally used [26].

The electrode reactions on a metal surface proceeding at a finite rate, involve the transport of reactive species between the electrode and the solution [27]. The three principle modes of ion transport from an electrode to an electrolyte are a) migration b) diffusion and c) convection; as per Eqn. 1 (given by the three terms on the right hand side of the equation) [27]. The rate of transfer for particular species $i$ thus has the following general form for a system at steady-state, with the species transported along a $Y$ direction:

$\mathrm{N}_{\mathrm{i}}=\mathrm{cU} \frac{\partial \Phi}{\partial \mathrm{y}}-\mathrm{D} \frac{\partial \mathrm{c}}{\partial \mathrm{y}}+\mathrm{Vc}$

Where: $\mathrm{N}_{\mathrm{i}}$ is the rate of transfer of species $\mathrm{i}$, in gram ions $/ \mathrm{cm}^{2} . \mathrm{sec}, \mathrm{c}$ is the concentration of the species, gram ions $/ \mathrm{cm}^{3}$, $\mathrm{U}$ is the mobility given by $\mathrm{cm}^{2} / \mathrm{sec}$-volt, $\mathrm{y}$ is the distance in the direction of transport, $\mathrm{cm}$, D is the diffusion coefficient, $\mathrm{cm}^{2} / \mathrm{sec}, \Phi$ is the solution potential in volts and $\mathrm{V}$ is the velocity of the bulk fluid movement in the direction of ion transport, $\mathrm{cm} / \mathrm{sec}$. In a stagnant electrolyte, the electrolyte flow velocity is zero, and thus the convection term may be neglected, provided the tip is rastered carefully enough not to promote electrolyte movement by itself. That is, scan rates must be adequately selected accounting for the tip dimensions and the tip-substrate separation. In the presence of excess supporting electrolyte, the migration is mainly confined to the supporting anions/cations, rather than the ions associated with the redox species utilised by the Pt tip [27]. When the Pt UME is placed in a solution containing a supporting electrolyte and an oxidizable/reducible species known as the redox mediator species (preferably a species undergoing a reversible one-electron transfer reaction), heterogeneous reaction occurs at the tip as the applied potential approaches its corresponding standard potential. Initially the currents emanating from the Pt tip are under kinetic control (see Figure 1 in the case of ferrocenemethanol oxidation at a Pt UME) [28], where the electrochemical current emanating from the electrode, varies exponentially with the applied potential. However, at increased polarisation, the electrochemical current attains a steady state diffusion limited current. At this point, 
the electrochemical current measured at the electrode, depends on the rate of diffusion of the electroactive species towards the electrode. This diffusion limited steady current is defined by the Cottrell equation, and is related to the bulk concentration of the electroactive species, and also the thickness of the diffusion layer (given by (Eqn. 2)) [29-30],

$I_{\text {lim }}=4 n F D a c_{\text {bulk }}$

where $\mathrm{n}$ is the number of electrons passed per reactant, $\mathrm{F}$ the Faraday constant, $\mathrm{D}$ and cbulk the diffusion coefficient and bulk concentration of the reactant, respectively; and $a$ is the radius of the microelectrode. Prior to spatial electrochemical micrographs being collected via SECM, an approach curve measurement is performed. Firstly, the Pt UME, is held at a fixed potential, in a known solution, where it undergoes some diffusion controlled electrochemical reaction (resulting in a constant diffusion limited current). The polarised Pt tip is then simultaneously moved along the $Z$ direction towards the sample surface. Once the Pt tip reached close to sample surface, depending on the nature of the substrate, fluctuations of current are recorded on the Pt tip. If the sample is conductive (such as a metal) it is capable of regenerating the reactant species producing a local increase in its concentration (positive feedback), whereas if the sample is insulating, the diffusion of the electroactive species is hindered (negative feedback). The approach curve thus serves to deduce the position of the sample (along the $Z$ axis) from the Pt tip. Several quantitative models have been developed to evaluate the kinetics of regeneration taking place on the sample surface, from the approach curve data [31-34].

The separation of effects of the surface topography and the electrochemical reactivity of the surface has been a long-standing challenge in high-resolution SECM [25]. The modern SECM has much advancement to overcome this challenge by firstly having various versions of tip position modulation such as shear-force based control, AC-SECM, integration with an AFM tip (AFM-SECM) and intermittent contact SECM [25,35]. The SECM tips have evolved with the development of submicrometre sized tips, nanoelectrodes, soft stylus microelectrodes and also the facilitation of batchproduction of nanoelectrodes as SECM tips [25,35]. The latest trend in SECM also involves the use of micro- and nanopipette-based probes. A nanopippette could be developed by pulling either a glass or quartz capillary and then filling with solution. The presence of this second solution, within the probe facilitates a wider set of experiments using such pipet-based tips, which could not have been performed using the conventional metallic tips [25]. Finally, the resolution of electrochemical imaging has also been enhanced regarding the separation of the contributions of surface topography and substrate reactivity with the recent development of the electron transfer/ion transfer mode of SECM (ET/IT SECM). This operation mode involves the delivery of reagent from an immiscible liquid phase using 
a nanopipette-based arrangement [36]. Electron transfer occurs at the substrate whereas ion transfer happens across the liquid-liquid interface, greatly diminishing diffusional broadening in the system.

\section{SECM: Amperometric modes of operation}

SECM can image a surface in the positive feedback mode, wherein, areas with different conductivity regenerate the electroactive species at different rates, resulting in a spatial electrochemical image of the surface. In negative feedback mode, diffusion of the electroactive species is hindered, and therefore one can image the topography of an insulating sample using SECM. The SECM can also be operated in redox competition mode (RC-SECM) mode [37]. In this mode, the Pt tip and the sample compete for reactions with same analyte in the solution. In the case of oxygen being the analyte, oxygen reduction at the Pt tip will lead to a constant current signal unless the tip is positioned above a competing $\mathrm{O}_{2}$ consuming site on the sample, which will locally alter the $\mathrm{O}_{2}$ concentration concomitantly leading up to a diminished current flow through the Pt tip [37]. Thus, the active sites on the sample for oxygen reduction could be deduced in this mode. This mode of operation would however, have no relevance to $\mathrm{Mg}$ on the basis that $\mathrm{O}_{2}$ plays no role in $\mathrm{Mg}$ corrosion [38], and only topographic information concerning the evolution of a corroding magnesium surface can be obtained [39]. Other modes of SECM imaging include the Sample Generation-Tip Collection (SG-TC) mode where some electroactive species released by the substrate are oxidised/reduced by the Pt tip, resulting in the associated current revealing the electrochemically active sites on the substrate. Fushimi et al. [40] measured hydrogen evolution from a Fe-3 at.\% Si alloy in 0.01 mol.dm ${ }^{-3} \mathrm{HCl}$ solution, by rastering a Pt tip (held at $0.2 \mathrm{~V}$ vs SHE to oxidise hydrogen) a few microns above the metal surface [40]. In the Tip Generation-Sample Collection (TG-SC) mode the tip releases some reactive species, which interact with the sample, allowing one to interpret the influence of a particular species on the sample [25]. These different modes of SECM operation are schematically shown in Figure 2.

\section{SECM on Mg using Pt amperometric tips}

In the context of Mg, SECM studies have been undertaken by several researchers using the conventional Pt microelectrode in amperometric operation [39,41-44]. This implies the selection of a suitable redox mediator for the monitoring of faradaic currents at the UME. The very negative reduction potential of magnesium prevents the metal ions dissolved during the corrosion processes to be electrochemically converted at the UME. For the same reason, redox species in the oxidized state 
cannot be added as artificial redox mediators for feedback operation since the magnesium substrate will act as a reducing agent. In addition, magnesium corrosion is accompanied by evolution of hydrogen gas, again a strong reducing agent in the system. Since decay of hydrogen oxidation only occurs at potentials more positive of $+0.2 \mathrm{~V}$ vs. $\mathrm{Ag} / \mathrm{AgCl}$, only redox mediators that are oxidized at potentials more positive than this value may be considered for SECM imaging. In practice, these facts restrict the range of available redox mediators to almost exclusively Fe(II)-based species, namely ferrocyanide [41] and ferrocenemethanol [43-44].

The redox competition mode also finds very limited application for the investigation of the corrosion of magnesium-based materials. It was mentioned before that oxygen plays a minor role in the corrosion of magnesium, thus only a small fraction of the dissolved oxygen in the proximity of the corroding substrate will be reduced on its surface, and therefore almost all the soluble molecule undergo chemical conversion at the Pt UME [39]. On this basis, only topographic imaging of the surface at different stages of corrosion progress with time can be attempted in situ. That is, the SECM was employed to visualize holes above the Mg surface after different different periods of immersion in the electrolyte. But even this procedure has limited applicability in an actively corroding metal surface due to the evolution of hydrogen gas, as the oxidation range of the later almost encompasses the potential range employed for the monitoring of oxygen at Pt (i.e., $-0.5 \geq \mathrm{E}_{\text {tip }} \geq-0.7 \mathrm{~V}$ vs. Ag/AgCl) [43]. Therefore, $\mathrm{O}_{2}$ reduction current would be masked by the greater oxidation current of $\mathrm{H}_{2}$. A different scenario is found in the case of physical separation between the cathodic and anodic sites in the system, as it occurs when a nobler metal is galvanically-coupled to magnesium. Thus, cathodic half-reaction would occur almost exclusively on the less active metal, and a redox competition towards oxygen reduction is established between the scanning tip and the cathodes on the substrate [45].

A prominent mode is the SG-TC mode, where the Pt tip is held at around $-0.05 \mathrm{~V}$ vs. Ag/ $\mathrm{AgCl}$ reference electrode, and thus could oxidise $\mathrm{H}_{2}$ bubbles emanating from the corroding Mg surface (3).

$\mathrm{H}_{2} \longrightarrow 2 \mathrm{H}^{+}+2 \mathrm{e}^{-}$

The Pt tip under such a potential when rastered across an Mg sample, can thus 'image' the cathodically active sites (hydrogen evolution sites) [42]. An example of such measurements on Mg alloys, has been shown in Figure 3 (adapted from Tefashe et al [42]). The microstructure of the sample is shown in Figure 3(I), and the corresponding SECM image when the Pt-tip (held at $-0.05 \mathrm{~V}$ vs. Ag/AgCl) is rastered across the Mg alloy surface, 5 micron above it, is shown in Figure 3(II). The SECM profile shows pits on the Mg surface (after 5 minutes), and high rates of hydrogen evolution after an hour. It is noted that a one-to-one correspondence between the $\mathrm{Mg}$ microstructure and hydrogen evolution 
from the surface cannot be directly viewed, and also the hydrogen oxidation currents detected by the Pt tip increase by two orders of magnitude after about an hour [42] The SECM image shows several active sites of localised corrosion on the Mg surface. Similar studies have recently been performed by Jamali et al. [43] on the Mg alloy AZ31, however it was difficult to link the one-to-one correspondence between the alloy microstructure, and the local electrochemical kinetics.

\section{Challenges in monitoring hydrogen evolution in SG-TC mode}

The cyclic voltammograms (CV) of Pt electrodes in acidic solutions show that at a potential of around $0 \mathrm{~V}$ vs. $\mathrm{Ag} / \mathrm{AgCl}$, the hydrogen oxidation reaction is strictly not a diffusion controlled process [46]. Thus, the hydrogen oxidation current recorded by the Pt tip, as it rasters along the Mg surface, may be partially affected by the local ionic concentration, and also the products of the electrode reaction $\left(\mathrm{H}^{+}\right)$, which increases in concentration in the locality, and could also get reduced back on the Pt surface (4).

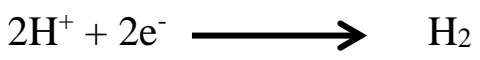

(Eqn. 4)

Such a scenario makes the quantification of the rate of hydrogen evolution from the Mg surface difficult (when compared to a scenario with a diffusion controlled electrode reaction). As Mg corrodes hydrogen bubbles form extensively across its surface. The fraction of the electrode surface covered by the adhering bubbles is defined as the (fractional) bubble coverage [47]. The bubbles insulate a fraction of the surface, and making it inactive and correspondingly increasing the actual current density emanating from the surface. These bubbles also contribute to an IR drop in the Mg corrosion cell [47]. The presence of gas bubbles in the Luggin capillary (connected to the reference electrode chamber) significantly disrupts the potential measurement of the electrode, showing "noise” like fluctuating data during measurement. Similarly the formation of hydrogen bubbles on the Mg surface disrupts the SECM measurements with sudden spikes of signals recorded by the Pt tip. The term stagnant needs to be cautiously applied in the case of corroding Mg systems. Hydrogen bubbles formed on the surface always exert motion on the liquid; as the bubble grows, it pushes the electrolyte (liquid) away in a radial direction [48-50]. Even when the bubbles detach from the surface, a compensating flow of the electrolyte towards the electrode surface occurs [48-50]. Thus the formation and growth of hydrogen bubbles intrinsically cause a deviation from stagnant conditions in the local electrolyte.

The bubbles are transported through the electrolyte by convection (depending on the density difference between the bubble and the electrolyte). An upward moving hydrogen bubble displaces as equal volume of the electrolyte, and thus contributes to a convection flux of species moving away from the 
Pt tip, and also a corresponding convection flux of hydrogen moving towards the Pt surface [48-50]. This convection flux may increase the currents measured by the Pt tip (and thus cause significant variations), while operating in the SG-TC mode over a corroding Mg surface. Since the rate of hydrogen evolution is not uniform across the Mg surface, hydrogen bubbles form at different rates, and thus of different sizes upon the Mg surface. Once these bubbles detach from the metal surface it moves upwards, and attains a terminal velocity, which depends on the bubble diameter, and other parameters like the density and viscosity of the solution (5) [51].

$v_{T}=\left(\frac{2 \rho g}{9 \eta}\right) r^{2}$

Where, $v_{T}$ is the terminal velocity in $\mathrm{cm} / \mathrm{s}, \rho$ is the density of the electrolyte in $\mathrm{gm} / \mathrm{cm}^{3}, \mathrm{~g}$ is $9.8 \mathrm{~cm} / \mathrm{sec}$, $\eta$ is the viscosity of the electrolyte in $\mathrm{cm}^{2} / \mathrm{sec}$, and $\mathrm{r}$ is the radius of the bubble in $\mathrm{cm}$. Bubbles with a larger radius thus move faster upwards, when compared to bubbles with a lower radius. This implies that bubbles may move towards the electrolyte/air interface at different rates (terminal velocities), which thus originates local micromixing of the electrolyte in the system (due to regions of different densities) resulting in local turbulence within the electrolyte. Hydrogen bubbling on the Mg surface could thus contribute to convective fluxes (summarised in Figure 4), which may perturb current measurements at the SECM tip.

Finally, the $\mathrm{H}^{+}$released into the electrolyte by the Pt tip during hydrogen oxidation, may cause significant local acidification in the vicinity of the Mg surface, and correspondingly increase the corrosion of Mg with exposure to the polarised Pt tip.

\section{SECM on Mg using Ion-Selective Electrodes}

Several researchers have performed local measurements on $\mathrm{Mg}$ using $\mathrm{Mg}^{2+}$ ion selective electrode systems [12,45,52-53]. Izquierdo and co-workers [45] developed $\mathrm{Mg}^{2+}$ ion-selective micro-tipped electrodes (ISME), which could be used in the SECM mode, and could locally detect $\mathrm{Mg}^{2+}$ ions emanating from the Mg surface. Such micro-tipped electrodes comprise of an $\mathrm{Mg}^{2+}$ ionophore cocktail behaving holistically as an ion-selective membrane. The $\mathrm{Mg}^{2+}$ ionophore (bis-N,N-dicyclohexylmalonamide) [54] in the cocktail, selectively promotes permeation of the $\mathrm{Mg}^{2+}$ ions through the membrane (ionophore cocktail). An internal reference electrode is positioned above this membrane, immersed an internal electrolyte such as $\mathrm{MgCl}_{2}$ with $\mathrm{KCl}$ (Figure 5). This reference electrode is connected to another external reference electrode ( $\mathrm{Ag} / \mathrm{AgCl}$ reference electrode) which is immersed 
in the analyte. When ions in the electrolyte make contact with the membrane, diffusion occurs from across both sides of the membrane. However, since the membrane selectively favours the permeation of $\mathrm{Mg}^{2+}$ ions (and not so much the anions) a potential gradient develops across the membrane. At equilibrium, known as the Gibbs-Donnan equilibrium, the potential difference across the membrane counterbalances the concentration gradient across the membrane, resulting in a zero net transport of ions across the membrane [55]. The membrane settles at a potential known as the Donnan potential. The internal reference electrode measures this potential versus external reference electrode. This equilibrium potential logarithmically varies with respect to the concentration of $\mathrm{Mg}^{2+}$ in the analyte/electrolyte. In a solid-contact ISE system, a carbon fiber coated with a conducting polymer (electropolymerized 3,4-ethylenedioxythiophene, PEDOT [56]) is immersed into the ionophore cocktail [53]. This conducting polymer element releases electrons to the carbon fiber depending on the membrane potential or the concentration of $\mathrm{Mg}^{2+}$ ions in the membrane (Figure 6). A different design for a solid-contact ISE system consists in coating gold with a conductive polymer (poly(3octylthiophene-2,5-diyl, POT) in contact with the $\mathrm{Mg}^{2+}$ ion-selective membrane at the apex tip of the micropipette [57]. The potential of the ISE versus the external reference electrode is calibrated to sense the local $\mathrm{Mg}^{2+}$ ion concentration in the electrolyte [48-49,51].

This technique or versions of this technique, have served to identify the local $\mathrm{Mg}^{2+}$ ion concentration, but a large quantity of the $\mathrm{Mg}^{2+}$ ions tend to migrate towards the cathodic sites, and form $\mathrm{Mg}(\mathrm{OH})_{2}$ [12]. Thus, the time resolution of these ISEs is critical, as a region with high concentration of $\mathrm{Mg}^{2+}$ ions may suddenly precipitate $\mathrm{Mg}(\mathrm{OH})_{2}$ causing a sudden drop in the $\mathrm{Mg}^{2+}$ ion concentration in the locality. It is also known that the anodes on the Mg surface may suddenly transform into cathodes (or dark regions) [10,58-61], and the $\mathrm{Mg}^{2+}$ local ion measurements must be supplemented with other experiments to identify the local electrochemical processes taking place on the metal surface.

Potentiometric operation can also be employed to monitor local $\mathrm{pH}$ variations in the solution in the proximity of the half-cell reactions on the surface. Both antimony- [45] and iridium-based [44] UME probes were employed to this end. Significant alkalization up to $10-11 \mathrm{pH}$ values was monitored around the cathodic sites [43,45], whereas proposals of local acidification around the anodic sites down to $\mathrm{pH} 5.5$ [45] is still regarded controversial at this stage. Despite the success of these studies, still some caution must be kept regarding the stability of the oxide films responsible for the potentiometric response of the probe towards $\mathrm{pH}$ in the case of either high corrosion activity of the magnesium substrate or prolonged experiments. Hydrogen released by the substrate may eventually reduce the oxide films formed on the tip, thus severely compromising their stability and the range of applications as to characterize magnesium corrosion. 


\section{SECM on Mg using AC modes}

It has been shown above that conventional SECM measurements are based on recording electrochemical signals related to the interaction with the surface of a redox species in the solution phase [19]. Electrochemical operation is amperometric, applying a potential to the UME, and recording the current of a diffusion-controlled reversible redox reaction at the probe as the measuring signal. Unfortunately, the need of a redox mediator in solution may affect the electrical state of the surface, because the electrochemical mediator can polarize the system. That is, the potential of the system is determined by the ratio between the two species involved in the redox couple, and can be determined by applying the Nernst equation [23]. Additionally, the possible interference of any of the redox species on the actual corrosion process under investigation cannot be discarded, particularly when thin surface films, such as those formed during the adsorption of corrosion inhibitors on metals, are involved [62]. This limitation of scanning electrochemical microscopy can be overcome by using frequency-dependent alternating current scanning electrochemical microscopy (AC-SECM) [23]. In this case, an oscillating potential perturbation is applied to the tip, and the AC current flowing at the tip is measured for different frequencies of the perturbation signal $[23,63]$. This feature strongly amplifies the possibilities of the SECM, since electrochemical data are also conditioned by the parameters of the voltage oscillation whereas it does not require the addition of electrochemical mediators. This feature is very attractive regarding the potential of AC modes for the investigation of magnesium corrosion, in addition to the expectation of a smaller interference of hydrogen evolution on the electrochemical response of the noble metal serving as probe. In this way, AC-SECM avoids the main sources of interference described for conventional amperometric SECM operation, or the hazards on the stability of the oxide films serving as dual amperometric/potentiometric probes. On the other side, the main limitation arises from the significantly smaller scan rates imposed by the need of recording a frequency spectra at each point in a scanning grid. As result, in practical situations, ACSECM images are recorded at a single frequency [43,63], thus missing the valuable electrochemical information contained in a frequency spectrum [24].

The alternating current response monitors variations in the local chemical activity of a surface [64], and both positive- and negative-type responses for the AC current may be found when approaching a substrate with the Pt UME depending on the electrochemical/electrical reactivity of the substrate, and this is strongly dependent on the frequency of the AC potential perturbation [65]. The origin of the effect is schematically shown in Figure 7. In AC-SECM a positive feedback-type response is observed during the approach to an electrically conducting sample and approaching an insulating sample results 
in a negative feedback-type response, allowing to distinguish conducting and non-conducting sites from one another [62]. Thus, if the tip electrode is placed within the near-field distance to the sample, a thin electrolyte film is formed between the surface and the electrode. As the sample blocks the current lines, the resistance increases. However, if the tip electrode is placed above a large conductive sample, the detected solution resistance may decrease. The current always chooses the least resistive pathway; the presence of an electrically conducting surface allows the re-direction of the current through the sample. Since it is an alternating current, no actual electron transfer across the solid/liquid interface has to occur. The lower resistance of the pathway through the sample compared to the surrounding solution causes the current to increase in a positive feedback-type behaviour.

The first attempt to use the AC modes of SECM to investigate the corrosion of magnesium was performed by Baril and co-workers [63]. Changes in the resistive component of impedance measured at a high frequency (namely $120 \mathrm{kHz}$ ), effectively minimizing the contribution of capacitive components to the overall current response, were employed to image the progressive formation of an insulating $\mathrm{MgO}$ layer on the pure metal during exposure to $0.5 \mathrm{M} \mathrm{Na}_{2} \mathrm{SO}_{4}$ according to Eqns. (6) and (7) [63],

$\mathrm{Mg}^{2+}+2 \mathrm{OH}^{-} \longrightarrow \mathrm{Mg}(\mathrm{OH})_{2}$

$\mathrm{Mg}(\mathrm{OH})_{2} \rightleftarrows \mathrm{MgO}+\mathrm{H}_{2} \mathrm{O}$

In this way, images were obtained with increasing coverages of the reactive metal surface by the oxide layer with the elapse of time, whereas highly localized reactive sites (possibly pores in the oxide or nucleation of corrosion pits) were detected at longer exposures when the oxide layer covered the entire Mg surface [63]. Another interesting outcome was the observation that these active zones were moving on the magnesium surface by comparing subsequent images. More recently, the effect of frequency was exploited by Jamali et al. [43] to study the resistive properties of the oxide layer formed on magnesium. Domains of different film conductivity were imaged using a rather low frequency of the AC signal, namely $1 \mathrm{kHz}$, whereas the capacitive characteristics of the surface layer were explored over a range of frequencies by placing the UME probe above selected locations on the metal surface [43].

\section{Suggestions for further work:}


The high rate of hydrogen bubbling from the Mg surface could significantly impact its SECM response, as discussed above. Rapid corrosion rates also imply that the surface is changing, perhaps drastically, in relatively short periods (i.e. akin to the times required for an $X-Y$ SECM scan) making SECM measurements comparatively difficult upon an $\mathrm{Mg}$ surface. It can be recommended that some inert organic (such as ethylene glycol) be mixed with the aqueous phase (i.e. and NaCl electrolyte), or perhaps an effective (but not chemically interfering) inhibitor. Ethylene glycol is conventionally used as a solvent in practise, to avoid hydrolysis of water [65]. This may aid in reducing corrosion, and the rate of hydrogen evolution from the Mg surface, for the purpose of SECM imaging, minimising the variation in the distribution/location of anodes and cathodes on the Mg surface with time, perhaps allowing to identify anodic and cathodic activity variations across the surface as it has proven to do in other systems. The topography of the surface also is prone to variations, as Mg oxides/hydroxides grow upon the surface and also may detach from the surface, thus affecting the tip-substrate distance in the SECM measurements. Advanced modes of SECM such as shear-force SECM [42] may serve to minimise the impact of the surface topography relative to surface reactivity.

\section{Summary}

Mg corrodes rapidly in aqueous solutions accompanied by high rates of hydrogen evolution from its surface. Secondary phases on the Mg surface, which are nominally cathodic relative to the matrix, initially act as the sites for hydrogen evolution, however with time, corroded or dark regions on the Mg surface may become local cathodes owing to the 'cathodic activation' of Mg. As the anodic regions on the Mg surface evolve with time into cathodes, particularly for pure or solid solution alloys, the anode/cathode distribution across the Mg surface can vary constantly with time. At cathodic sites, $\mathrm{Mg}^{2+}$ ions precipitate as $\mathrm{Mg}(\mathrm{OH})_{2}$ (aided by an alkaline $\mathrm{pH}$ ). Therefore there is a constant variation in the local electrolyte and the electrode surface complicating SECM studies upon Mg.

SECM can be performed via the use of metallic microelectrodes (such as the Pt UME) or micro-pipette electrodes. In the case of Mg, SECM has been carried out using the Pt UME, in the SG-TC mode, where hydrogen bubbles emanating from the Mg surface are immediately oxidised by the Pt (held at around $-0.05 \mathrm{~V}$ vs $\mathrm{Ag} / \mathrm{AgCl})$. The corresponding currents recorded by the Pt tip, should ideally correspond to the cathodically active sites on the Mg surface. However, from SECM a one-to-one correspondence between the microstructure of the $\mathrm{Mg}$ surface, and the associated electrochemical response is difficult to validate. The hydrogen oxidation currents measured by the Pt tip, vary greatly (over an order of magnitude) following an hour of exposure. Hydrogen bubbles formed on the Mg 
surface make regions of the surface 'inactive' and formation/detachment of such bubbles also result in local convective fluxes. Since hydrogen evolution on $\mathrm{Mg}$ varies across the surface, the size of the bubbles emanating from surface is inconsistent, resulting in different velocities of the bubbles through the electrolyte - resulting in micromixing within the electrolyte which affects SECM measurements. Hydrogen oxidation is not a diffusion controlled reaction on the Pt tip, and therefore, the currents on the Pt tip may also vary from its own reactions, rather than from the Mg surface beneath. Finally, the $\mathrm{H}^{+}$ions released from the Pt tip may contribute to local acidification close to the Mg surface, which may alter its dissolution.

$\mathrm{Mg}^{2+}$ ion selective micro-pipette electrodes (ISE) have been used as the SECM tip for electrochemically imaging a corroding Mg surface. The ionophore cocktail within the ISE acts as a membrane for permeation of $\mathrm{Mg}^{2+}$ - such that when the ISE is exposed to the electrolyte in the vicinity of the $\mathrm{Mg}$ surface, diffusion/migration of the $\mathrm{Mg}^{2+}$ take place through this membrane eventually attaining the Gibbs-Donnan equilibrium. The membrane attains a corresponding potential known as the Donnan potential, which is measured with respect to an external reference electrode immersed in the same electrolyte. This potential varies logarithmically with the concentration of $\mathrm{Mg}^{2+}$ ions in the electrolyte. The ISEs may be a liquid contact ISE, wherein, the internal solution within the ISE is $\mathrm{MgCl}_{2}$ with $\mathrm{KCl}$, or a solid contact ISE which uses a carbon fiber coated with PEDOT, to measure variations in the Donnan potential of the membrane due to the local $\mathrm{Mg}^{2+}$ ions concentration in solution. This technique may image local $\mathrm{Mg}^{2+}$ ion concentrations, but other modes of SECM may also be used to achieve a holistic analysis of a corroding Mg surface. To achieve high resolution SECM images of the surface, the corrosion rate of $\mathrm{Mg}$, and also the rate of hydrogen bubbling from it need to be reduced. The use of organic non-aqueous solvents to dilute any aqueous electrolyte could serve to mitigate this issue. The potential of AC modes for studying the corrosion mechanism still needs to be addressed, though it appears an attractive possibility at this stage.

\section{References}

1) I. J. Polmear, Light Alloys, fourth edition: From Traditional Alloys to Nanocrystals, Chapter 5, 2006, Butterworth-Heinemann.

2) G. L. Makar, J. Kruger, International Material Reviews, 1993, 38, 138.

3) Cain 2014, Corrosion.

4) N. T. Kirkland, J. Lespagnol, N. Birbilis, M. P. Staiger, Corrosion Science, 2010, 52, 287.

5) N. T. Kirkland, G. Williams, N. Birbilis, Corrosion Science, 2012, 65, 5.

6) R. E. McNulty, J. D. Hanawalt, Transactions of the Electrochemical Society, 1942, 423.

7) M. Danaie, R. M. Asmussen, P. Jakupi, D. W. Shoesmith, G. A. Botton, Corrosion Science, 2013, 77, 151. 
8) D. S. Gandel, M. A. Easton, M. A. Gibson, T. Abbot, N. Birbilis, Corrosion Science, 2014, 81, 27.

9) A. D. Sudholz, N. T. Kirkland, R. G. Buchheit, N. Birbilis, Electrochemical and Solid State Letters, 2011, 14, C5.

10) G. Williams, N. Birbilis, H. N. McMurray, Electrochemistry Communications, 2013, 36, 1.

11) G. Williams, H. N. McMurray, Journal of the Electrochemical Society, 2008, 155, C340.

12) S. V. Lamaka, O. V. Karavai, A. C. Bastos, M. L. Zheludkevich, M. G. S. Ferreira, Electrochemistry Communications, 2008, 10, 259.

13) Y. González-García, G. T. Burstein, S. González, R. M. Souto, Electrochemistry Communications, 2004, 6, 637.

14) A. M. Simões, A. C. Bastos, M. G. Ferreira, Y. González-García, S. González, R. M. Souto, Corrosion Science, 2007, 49, 726.

15) S. González, J. J. Santana, Y. González-García, L. Fernández-Mérida, R. M. Souto, Corrosion Science, 2011, 53, 1910.

16) Y. González-García, J. M. C. Mol, T. Muselle, I. De Graeve, G. Van Assche, G. Scheltjens, B. Van Mele, H. Terryn, Electrochemistry Communications, 2011, 13, 169.

17) E. Martinez-Lombardia, Y. Gonzalez-Garcia, L. Lapeire, I. De Graeve, K. Verbeken, L. Kestens, J. M. C. Mol, H. Terryn, Electrochimica Acta, 2014, 116, 89.

18) D. Mandler, Scanning Electrochemical Microscopy, Chapter 15, Second Edition, edited by A. J. Bard and M. V. Mirkin, 2012, CRC Press.

19) A. J. Bard, F. R. F. Fan, J. Kwak, O. Lev, Analytical Chemistry, 1989, 61, 132.

20) F.-R- F. Fan, C. Demaille, Scanning Electrochemical Microscopy, Chapter 3, Second Edition, edited by A. J. Bard and M. V. Mirkin, 2012, CRC Press.

21) G. Denuault, G. Nagy, K. Toth, Scanning Electrochemical Microscopy, Chapter 10, Second Edition, edited by A. J. Bard and M. V. Mirkin, 2012, CRC Press.

22) B. Horrocks, M. V. Mirkin, D. T. Pierce, A. J. Bard, G. Nagy, K. Toth, Analytical Chemistry, 1993, 65, 1213.

23) K. Eckhard, W. Schuhmann, Analyst, 2008, 133, 1486.

24) V. Kuznetsov, A. Maljusch, R. M. Souto, A. S. Bandarenka, W. Schuhmann, Electrochemistry Communications, 2014, 44, 38.

25) M. V. Mirkin, W. Nogala, J. Velmurugan, Y. Wang, Physical Chemistry Chemical Physics, 2011, 13, 21196.

26) C. Demaille, F.-R. F. Fan, Scanning Electrochemical Microscopy, Chapter 3, Second Edition, edited by A. J. Bard and M. V. Mirkin, 2012, CRC Press.

27) C. W. Tobias, M. Eisenberg, C. R. Wilke, Journal of the Electrochemical Society, 1952, 99, 359C.

28) S. Thomas, Towards Developing Self-repairing Oxides to Protect Zinc, Chapter 5, 2013, PhD thesis.

29) K. B. Oldham, J. C. Myland, Fundamentals of Electrochemical Science, Chapter 7, 1994, Academic Press.

30) T. Z. Fahidy, Principles of Electrochemical Reactor Analysis, Chapter 1, 1985, Elsevier.

31) J. L. Amphlett, G. Denuault, Journal of Physical Chemistry B 1998, 102, 9946.

32) C. Lefrou, Journal of Electroanalytical Chemistry, 2006, 592, 103.

33) R. Cornut, S. Griveau, C. Lefrou, Journal of Electroanalytical Chemistry, 2010, 650, 55.

34) R. Cornut, A. Bhasin, S. Lhenry, M. Etienne, C. Lefrou, Analytical Chemistry, 2011, 83, 9669.

35) C. Kranz, Analyst, 2014, 139, 336.

36) Y. Wang, K. Kececi, J. Velmurugan, M. V. Mirkin, Chemical Science, 2013, 4, 3606.

37) K. Eckhard, W. Schuhmann, Electrochimica Acta, 2007, 53, 1164.

38) G.-L. Song, Corrosion of Magnesium Alloys, Chapter 1, edited by G.-L. Song, 2011, Woodhead.

39) M. D. Pereda, C. Alonso, M. Gamero, J. A. del Valle, M. Fernández Lorenzo de Mele, Materials Science and Engineering C, 2011, 31, 858. 
40) K. Fushimi, K. A. Lill, H. Habazaki, Electrochimica Acta, 2007, 52, 4246.

41) X. Liu, T. Zhang, Y. Shao, G. Meng, F. Wang, Corrosion Science, 2009, 51, 1772.

42) U. M. Tefashe, M. E. Snowden, P. D. Ducharme, M. Danaie, G. A. Botton, J. Mauzeroll, Journal of Electroanalytical Chemistry, 2014, 720-721, 121.

43) S. S. Jamali, S. E. Moulton, D. E. Tallman, M. Forsyth, J. Weber, G. G. Wallace, Corrosion Science, 2014, 86, 93.

44) W. Liu, F. Cao, Y. Xia, L. Chang, J. Zhang, Electrochimica Acta, 2014, 132, 377.

45) J. Izquierdo, L. Nagy, I. Bitter, R. M. Souto, G. Nagy, Electrochimica Acta, 2013, 87, 283.

46) D. Zhan, J. Velmurugan, M.V. Mirkin, Journal of the American Chemical Society, 2009, 131, 14756.

47) H. Vogt, R. J. Balzer, Electrochimica Acta, 2005, 50, 2073.

48) N. Ibl, Chemie Ingenieur Technik, 1961, 33, 69.

49) H. Vogt, Chemie Ingenieur Technik, 1980, 52, 418.

50) H. Vogt, Electrochimica Acta, 1993, 38, 1421.

51) M. Yersel, The Physics Teacher, 1991, 335.

52) R. M. Souto, A. Kiss, J. Izquierdo, L. Nagy, I. Bitter, G. Nagy, Electrochemistry Communications, 2013, 26, 25.

53) J. Izquierdo, A. Kiss, J. J. Santana, L. Nagy, I. Bitter, H. S. Isaacs, G. Nagy, R. M. Souto, Journal of the Electrochemical Society, 2013, 160, C451.

54) K. Toth, E. Lindner, M. Horvath, J. Jeney, E. Pungor, I. Bitter, B. Agai, L. Toke, Electroanalysis, 1993, 5, 781.

55) K. N. Mikhelson, Ion Selective Electrodes, Lecture Notes in Chemistry, Volume 81, Springer.

56) R.M. Souto, J. Izquierdo, J.J. Santana, A. Kiss, L. Nagy, G. Nagy, Current Microscopy Contributions to Advances in Science and Technology, p. 1407, edited by A. Méndez-Vilas, 2012, Formatex Research Center.

57) S. V. Lamaka, M. G. Taryba, M. L. Zheludkevich, M. G. S. Ferreira, Electroanalysis, 2009, 21, 2447.

58) G. S. Frankel, A. Samaniego, N. Birbilis, Corrosion Science, 2013, 70, 104.

59) M. Curioni, Electrochimica Acta, 2014, 120, 284.

60) M. Taheri, J. R. Kish, N. Birbilis, M. Danaie, E. A. McNally, J. R. McDermid, Electrochimica Acta, 2014, 116, 396.

61) N. Birbilis, A. D. King, S. Thomas, G. S. Frankel, J. R. Scully, Electrochimica Acta, 2014, 132, 277.

62) B. H. Horrocks, D. Schmidtke, A. Heller, A. J. Bard, Analytical Chemistry, 1993, 65, 3605.

63) G. Baril, G. Galicia, C. Deslouis, N. Pébère, B. Tribollet, V. Vivier, Journal of the Electrochemical Society, 2007, 154, C108.

64) M. A. Alpuche-Aviles, D. O. Wipf, Analytical Chemistry, 2001, 73, 4873.

65) M. Pähler, J.J. Santana, W. Schuhmann, R.M. Souto, Chemistry - A European Journal, 2011, 17, 905.

66) W. Li, T. Cochell, A. Manthiram, Scientific Reports, 2013, 3, 1229. 


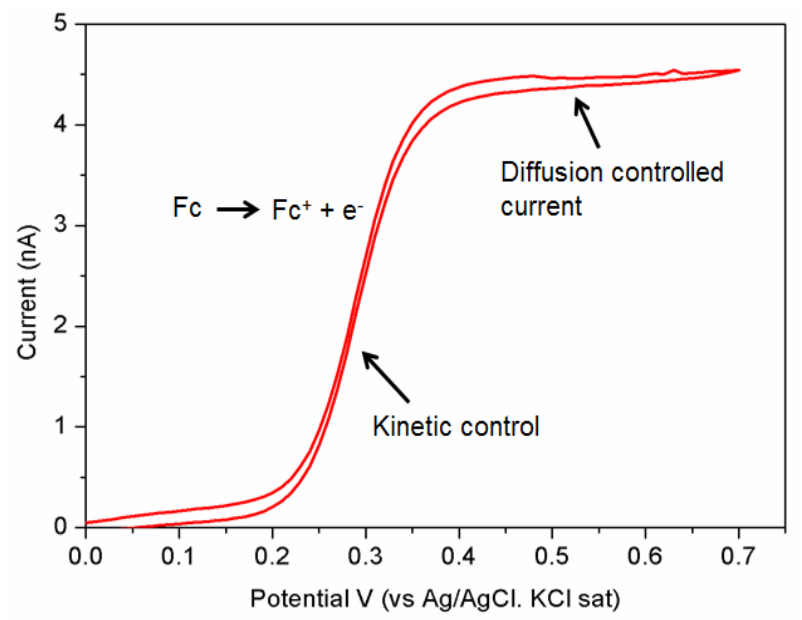

Figure 1: (Adapted from [28]) Cyclic voltammogram of a Pt microelectrode in a 5mM Ferrocenomethanol (with $0.05 \mathrm{M} \mathrm{NaNO}_{3}$ ) solution. Ferrocenomethanol undergoes oxidation to form the Ferrocenium ion.
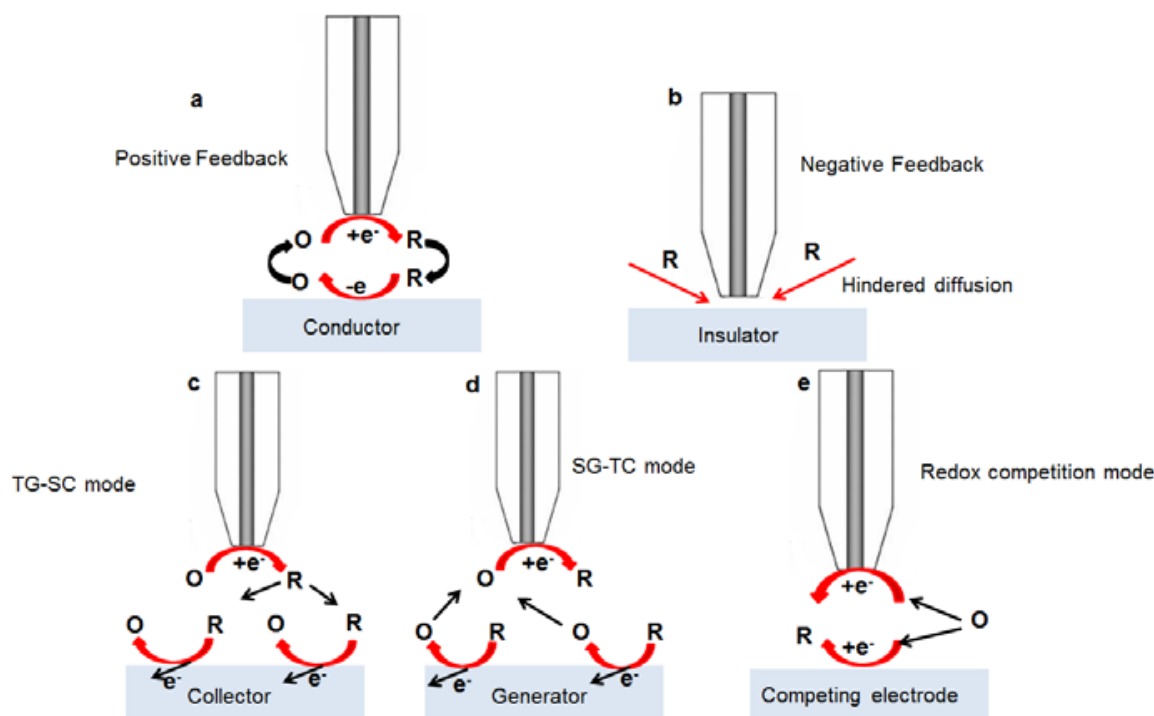

Figure 2: (Adapted from [18]) The different modes of conventional amperometric SECM operation: a) positive feedback, b) negative feedback, c) redox competition, d) Tip GenerationSample Collection (TG-SC), and e) Sample Generation-Tip Collection (SG-TC) mode. 

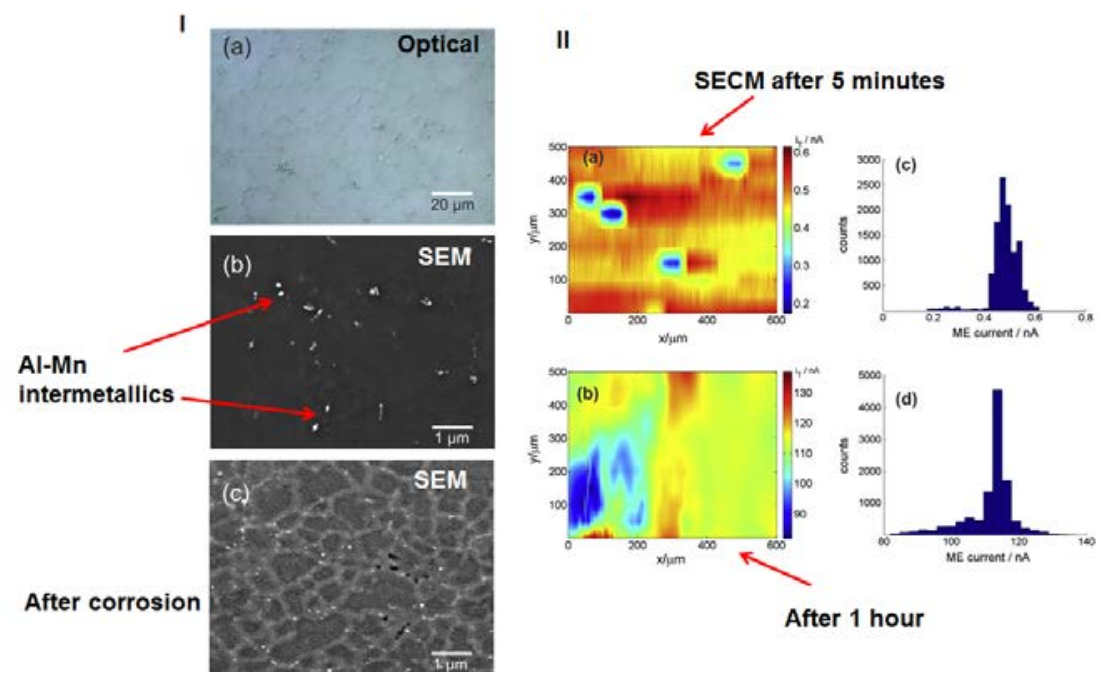

Figure 3: (Adapted from Tefashe et al [42]) I. a) Optical image of the AM-50 alloy, b) SEM image showing the Al-Mn intermetallics and c) SEM image of the surface after exposure. II. SECM image of the AM-50 alloy, exposed to $0.6 \mathrm{M} \mathrm{NaCl}$ solution, in the SG-TC mode (where hydrogen evolved from the Mg surface is oxidised at the Pt tip); a) After 5 minutes of exposure b) after 1 hour of exposure c) and d) histogram representations of currents generated from a) and b) respectively.

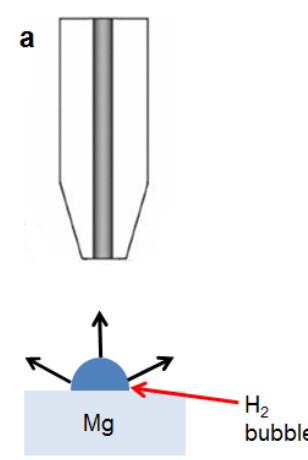

Radial force exerted during growth of the bubble
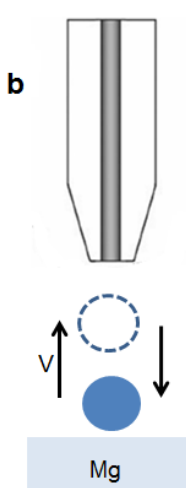

Bubble detaches and moves with a velocity " $v$ ", and displaces an equal volume of electrolyte

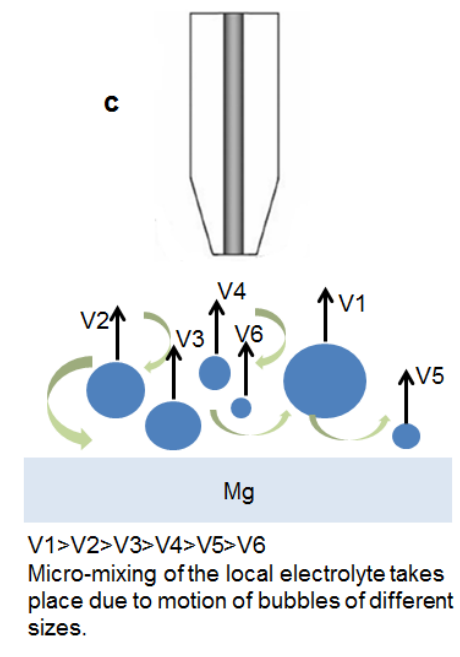

Figure 4: Different sources of convective fluxes, in a layer of local electrolyte close to the Mg surface. a) Due to the radial force exerted by the hydrogen bubble during its growth, b) Due to motion of the bubble with a velocity ' $v$ ', displacing a corresponding volume of the electrolyte, hydrogen bubble moves towards the Pt tip with a velocity ' $v$ ', whereas the electrolyte (with ions) moves away from the Pt electrode with the same velocity ' $v$ '. c) Micromixing within the electrolyte as bubbles of different sizes detach from the Mg surface; bubbles with larger radius have a higher terminal velocities. 

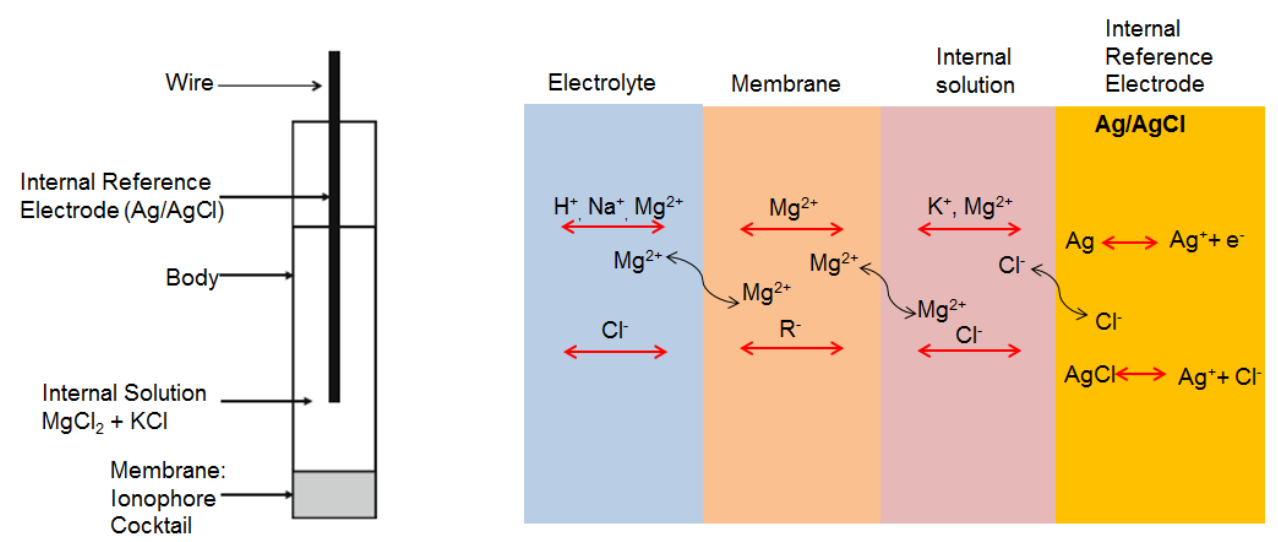

Figure 5: (Adapted from [44,54]). Left: Constituents of a $\mathrm{Mg}^{2+}$ ion selective electrode with liquid contact. Right: Process taking place between the different active components within a liquidcontact ion selective electrode system. The membrane sandwiched between the internal solution and the electrolyte supports $\mathrm{Mg}^{2+}$ ion transport and eventually attains an equilibrium potential (Donnan potential), which varies logarithmically with the $\mathrm{Mg}^{2+}$ ion concentration in the electrolyte. This potential is sensed by the internal reference electrode. The internal reference when connected to an external reference electrode, immersed in the same electrolyte "senses" the $\mathrm{Mg}^{2+}$ ion concentration.
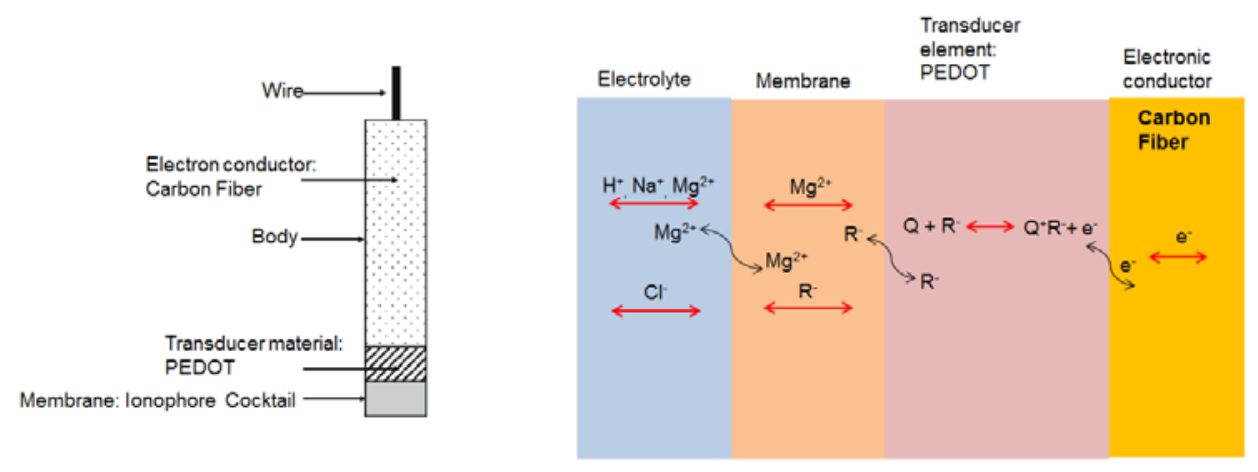

Figure 6: (Adapted from [52,54]). Left: Constituents of a $\mathrm{Mg}^{2+}$ ion selective electrode with solid contact. Right: Process taking place between the different active components within a solid-contact ion selective electrode system The membrane sandwiched between the transducer element and the electrolyte attains an equilibrium potential (Donnan potential), which varies logarithmically with the $\mathrm{Mg}^{2+}$ ion concentration in the electrolyte. The transducer element emits/receives electrons from the carbon fiber, to facilitate the equilibrium. The carbon fiber when connected to a reference electrode, immersed in the same electrolyte "senses" the $\mathrm{Mg}^{2+}$ ion concentration. 


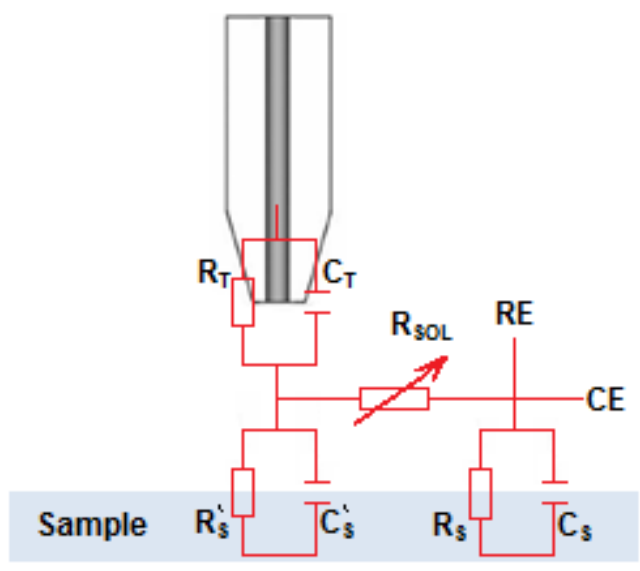

Figure 7: (Adapted from [61]). The alternating current mode of SECM. $\mathrm{R}_{\mathrm{T}}$ and $\mathrm{C}_{\mathrm{T}}$ are the resistance and capacitance of the tip-electrolyte interface, $\mathrm{R}_{\mathrm{S}}$ and $\mathrm{Cs}_{\mathrm{s}}$ are the resistance and capacitance of the sample-electrolyte interface and Rs' and Cs' the same at the site located just below the tip. Rsol is the ohmic resistance of the electrolyte between the UME and the counter electrode. 